support from: Bayer, O. Distler Grant/research support from: Actelion, Bayer, BiogenIdec, Boehringer Ingelheim, Chemo$\mathrm{mAb}$, espeRare foundation, Genentech/Roche, GSK, Inventiva, Lilly, medac, MedImmune, Mitsubishi Tanabe Pharma, Pharmacyclics, Novartis, Pfizer, Sanofi, Sinoxa and UCB, 2, Actelion, Bayer, BiogenIdec, Boehringer Ingelheim, ChemomAb, espeRare foundation, Genentech/Roche, GSK, Inventiva, Lilly, medac, MedImmune, Mitsubishi Tanabe Pharma, Pharmacyclics, Novartis, Pfizer, Sanofi, Sinoxa and UCB

\section{MESENCHYMAL STEM CELL-DERIVED EXTRACELLULAR VESICLES: A NOVEL THERAPEUTIC OPTION IN SYSTEMIC SCLEROSIS}

${ }^{1} \mathrm{P}$ Rozier, ${ }^{1} \mathrm{M}$ Maumus, ${ }^{2} \mathrm{~A}$ Maria, ${ }^{3} \mathrm{~K}$ Toupet, ${ }^{4} \mathrm{C}$ Jorgensen, ${ }^{2} \mathrm{P}$ Guilpain, ${ }^{1} \mathrm{D}$ Noel ${ }^{*} .{ }^{1} \mathrm{Inserm}$; ${ }^{2}$ Médecine Interne et Maladies Multi-Organiques, CHU Montpellier; ${ }^{3}$ Montpellier University; ${ }^{4}$ Clinical immunology and osteoarticular diseases Therapeutic Unit, CHU Montpellier, Montpellier, France

\subsection{6/annrheumdis-2018-EWRR2018.21}

Introduction Systemic sclerosis (SSc) is a rare intractable autoimmune disease, with unmet medical need. Cell therapy using mesenchymal stem cells (MSC) is a promising approach, and we recently reported its efficacy in a murine model of SSc induced by hypochlorite $(\mathrm{HOCl})$. Since MSC act primarily through the secretion of soluble factors released within extracellular vesicles (EV), the use of EV instead of cells seems an attractive alternative. Herein, we compared the effects of two types of $\mathrm{EV}$, exosomes and microparticles, in $\mathrm{HOCl}$-induced SSc.

Objectives Herein, we compared the effects of two types of $\mathrm{EV}$, exosomes and microparticles, in HOCl-induced SSc.

Methods $\mathrm{BALB} / \mathrm{c}$ mice were challenged with daily intradermal $\mathrm{HOCl}$ injections during 6 weeks to induce SSc. Each group was treated at mid-experiment with infusions of $2.5 \times 10^{5}$ murine MSC, $250 \mathrm{ng}$ of exosomes or microparticles isolated from IFN $\gamma$-activated or non-activated (NA) MSC. We measured skin thickness every week. At euthanasia (d42), we analysed the expression of fibrotic and inflammatory markers (collagens 1 and 3, $\alpha$ Sma, TGF $\beta$, MMP 1 and 9, TIMP1, IL1 $\beta$, IL6, TNF $\alpha$ ) in lungs and skin samples using RT-qPCR.

Results Mice treated with each subtype of EV displayed lower clinical scores, less histological lesions, lower expression of fibrotic and inflammatory markers, with enhanced expression of remodelling parameters in skin and lung tissues. The observed effects were similar to those obtained with MSC. No difference was noted between NA and IFN $\gamma$-activated EV.

Conclusions MSC-derived EV display potent antifibrotic properties in murine SSc. This new acellular therapy represents a promising approach in this disease.

Disclosure of interest None declared
0022 FOR EACH HLA-DRB1 GENOTYPE, THE LIKELIHOOD TO DEVELOP RA CORRELATES WITH THE PROBABILITY OF BINDING AT LEAST A PEPTIDE FROM PAD4

${ }^{1,2} \mathrm{~N}$ Balandraud, ${ }^{2}$ Auger, ${ }^{3,4} \mathrm{G}$ Texier, ${ }^{1,5} \mathrm{~J}$ Roudier*. ${ }^{1}$ Rheumatology, Hôpital Sainte Marguerite; ${ }^{2}$ UMRs 1097 Immunogénétique des maladies auto immunes, INSERM and Aix Marseille University; ${ }^{3}$ Centre d'épidémiologie et de santé publique des armées (CESPA), CESPA; ${ }^{4}$ UMR 912 - SESSTIM, INSERM Aix Marseille University; ${ }^{5}$ UMRs 1097 Immunogénétique des maladies auto immunes, INSERM and Aix Marseille University, Marseille, France

\subsection{6/annrheumdis-2018-EWRR2018.22}

Introduction The production of IgG ACPA can be helped by $\mathrm{T}$ lymphocytes specific for PADs, the enzymes that transform arginin into citrullin. Thus, the molecular basis for the HLADRB1 association with RA might be the capability for the two alleles encoded by a given HLA-DRB1 genotype to bind PAD4 derived peptides. ${ }^{1}$ We recently published a table showing that the relative risk to develop ACPA positive RA for the 106 most common genotypes. ${ }^{2}$ French HLA-DRB1 genotypes varies from 28 to 0.2 . For a given HLA-DRB1 genotype, the risk to develop RA should be correlated with the probability for the two HLA-DRB1 molecules encoded by this genotype to bind peptides from PAD peptides.

Objectives To test whether the risk of developing ACPA positive RA for each HLA-DRB1 genotypes correlates with the probability for the 2 HLA-DR alleles encoded by each genotype to bind at least one PAD4 peptide.

Methods 65 Synthetic peptides (20 mers) of human PAD4 and 167 peptides encompassing the $\mathrm{A}$ and $\mathrm{B}$ chains of human fibrinogen were synthesised in solid phase. Whenever there was an arginine residue, both the arginine and the citrullin variant were synthesised. In the end, we had 25 fibrinogen peptides containing neither arginin nor citrulline, 71 citrullinated and 71 arginine peptides from fibrinogen.

HLA-DRB1 peptide binding studies were performed by adding one microgram of purified HLA-DRB1 to ELISA wells coated with 10 micrograms PAD peptide. Bound HLA-DR was revealed by biotinylated anti HLA-DR antibody followed by peroxidase conjugated avidin.

Statistical analyses Correlation between HLA-DRB1 genotypic risk for RA and Likelihood to bind PAD4 for a given genotype was evaluated by Spearman's

Results HLA-DRB1 genotypic risks to develop RA correlate with likelyhood to bind PAD4 peptides ( $p=0.06$, Pearson's), not citrullinated Fibrinogen peptides $(p>0.6$ and $p>0.9)$.

Conclusions HLA-DRB1 genotypes are associated with a risk to develop RA and a likelihood to bind at least one of 65 overlapping PAD4 peptides. The strong correlation between these two parameters suggest that PAD4 peptide binding to HLA-DRB1 may be the basis of the HLA-DRB1 RA association. Such correlation is not observed when testing the binding of citrullinated or native peptides from Fibrinogen to HLA-DRB1 molecules. 